\title{
Chromogranin A as a marker of neuroendocrine neoplasia: an Italian Multicenter Study
}

\begin{abstract}
Maria Chiara Zatelli, Mirella Torta ${ }^{1}$, Antonette Leon $^{2}$, Maria Rosaria Ambrosio, Massimo Gion ${ }^{3}$, Paola Tomassetti ${ }^{4}$, Filippo De Braud ${ }^{5}$, Gianfranco Delle Fave ${ }^{6}$, Luigi Dogliotti ${ }^{1}$, Ettore $C$ degli Uberti, On behalf of the Italian CromaNet Working Group
\end{abstract}

Section of Endocrinology, Department of Biomedical Sciences and Advanced Therapies, University of Ferrara, Via Savonarola 9, 44100 Ferrara, Italy

${ }^{1}$ Medical Oncology Unit, University of Torino at S. Luigi Hospital, Orbassano, Torino, Italy

${ }^{2}$ ABO Association c/o Regional Centre for the Study of Biological Markers of Malignancy, General Regional Hospital, Venezia, Italy

${ }^{3}$ Regional Centre for the Study of Biological Markers of Malignancy, General Regional Hospital, Venezia, Italy

${ }^{4}$ Department of Internal Medicine and Gastroenterology, University of Bologna, Bologna, Italy

${ }^{5}$ Unit of Clinical Pharmacology and New Drugs, European Institute of Oncology (IEO), Milano, Italy

${ }^{6}$ Department of Gastroenterology, University of Rome at S. Andrea Hospital, Roma, Italy

(Requests for offprints should be addressed to E C degli Uberti; Email: dut@unife.it)

\begin{abstract}
Elevated circulating chromogranin $\mathrm{A}(\mathrm{CgA})$ levels are found in neuroendocrine tumors (NETs), but the diagnostic usefulness of this marker is still debatable. To assess the role of $\mathrm{CgA}$ for the diagnosis of gastroenteropancreatic (GEP) NETs and the identification of metastatic patients, an Italian multicenter observational study has been performed. CgA was evaluated in 202 GEP NET patients by IRMA and ELISA. The cutoffs for diagnosis and presence of metastases were identified by receiver-operating characteristic $(\mathrm{ROC})$ curve. We found good correlation between IRMA and ELISA. The ROC analysis identified a cutoff of $53 \mathrm{ng} / \mathrm{ml}$ for IRMA and $16 \mathrm{U} / \mathrm{l}$ for ELISA as discriminating between controls and patients with active disease (sensitivity 71.3 and $84 \%$; specificity 71 and $85 \%$ respectively). Metastases were present in 123 patients, having significantly higher CgA levels than patients without metastases. ROC analysis identified a cutoff of $146 \mathrm{ng} / \mathrm{ml}$ for IRMA and $67.3 \mathrm{U} / \mathrm{l}$ for ELISA as discriminating between patients with and without metastases (sensitivity 57 and $63.3 \%$; specificity 55.6 and $71.4 \%$ respectively). For pancreatic NETs positive and negative predictive values were 84 and $78 \%$ respectively (90\% specificity and $68 \%$ sensitivity). We found lower CgA levels in patients with extensive metastatic spread than in those with liver metastases only. These data assess the role of CgA evaluation in GEP NETs, and demonstrate that higher CgA levels associate with metastatic disease, confirming that $\mathrm{CgA}$ levels can provide a helpful practical biochemical marker for the clinical management of NETs, but with low sensitivity and specificity.
\end{abstract}

Endocrine-Related Cancer (2007) 14 473-482

\section{Introduction}

Chromogranin $\mathrm{A}(\mathrm{CgA})$ is an acidic glycoprotein expressed in the secretory granules of most normal and neoplastic neuroendocrine (NE) cell types, where it is released together with peptide hormones and biogenic amines (Taupenot et al. 2003). Elevated circulating CgA levels have been demonstrated in serum or plasma of patients with various NE tumors (NETs; Nobels et al. 1997, Guignat et al. 2001, Tomassetti et al. 2001).
Previous studies reported different ranges of sensitivity and specificity for circulating $\mathrm{CgA}$, according to histological characteristics of the tumor and to disease spread. These parameters have been demonstrated to depend also on the method used for serum or plasma $\mathrm{CgA}$ determination and on the threshold considered as pathologic (Schürmann et al. 1992, Stridsberg et al. 1995, Nobels et al. 1997, Baudin et al. 2001, Stivanello et al. 2001, Tomassetti et al. 2001). In order to clarify this 
issue, an Italian multicenter observational study has been performed in a large series of gastroenteropancreatic (GEP) NET patients and healthy controls to assess the usefulness of $\mathrm{CgA}$ determination for the diagnosis of sporadic GEP NETs and to establish the best cutoff value for the diagnosis of GEP NETs using the method of the receiver-operating characteristic (ROC) curve.

\section{Materials and methods}

\section{Subjects}

The control group was composed of 129 healthy individuals (61 males and 68 females; aged 44.2 \pm 8.4 years (mean \pm s.D.); range $22-59$ years) without evidence of NETs, malignancies, hypertension, renal or liver failure, and not treated with proton pump inhibitors.

Overall, 273 patients with NETs were enrolled between April 2003 and October 2004 in 40 different Italian centers, participating to the CROMaNET study, a multicenter observational study for the evaluation of $\mathrm{CgA}$ as marker for diagnosis and follow-up of NET. Among these subjects, 202 patients (109 males and 93 females; aged $58.5 \pm 13.8$ years; range $14-84$ years) were diagnosed from 1 to 120 months earlier (median 45 months) with GEP NET, pathologically proven by histological and immunohistochemical diagnosis after surgery or biopsy of primary tumor or metastases.

Exclusion criteria were kidney defect (plasma creatinine $>120 \mu \mathrm{M} / \mathrm{l}$; Canale \& Bravo 1994), liver failure, treatment with proton pump inhibitors, Parkinson's disease, pregnancy, or the presence of any other malignancy.

The GEP NET group included 73 patients with primitive NET site in the pancreas, 2 in the esophagus, 27 in the stomach, 7 in the duodenum, 71 in the ileum, 14 in the colon, and 8 in the rectum. Conventional imaging (abdominal and thoracic CT and/or MRI), as well as ultrasonography, endoscopy, echo-endoscopy, and somatostatin receptor scintigraphy (Octreoscan) were used for staging when appropriate. Among these patients, $123(60.9 \%)$ presented with metastases. The extent of metastatic spread was defined as locally advanced (when limited to regional lymph nodes), with liver metastases (when only liver metastases were evident) and with liver and extra-hepatic metastases (when bone, lung, or brain metastases were demonstrated). Patients were divided into four groups:

1) new diagnosis (ND, 81 patients): including patients diagnosed at the centre for the first time as having a GEP NET, with evidence of disease at study entry;
2) relapse (RL, 27 patients): patients with evidence of recurrent disease, not medically treated for at least 6 months before study entry;

3) stable disease (SD, 49 patients): patients with evidence of persistent disease, medically treated for at least 6 months before study entry;

4) remission (RM, 45 patients): patients previously treated (either surgically or medically) for a GEP NET, with no evidence of disease at study entry.

\section{CgA determination}

All samples were collected after an overnight fast, as previously described (Leon et al. 2005), for plasma and serum, both aliquoted and stored at $-80{ }^{\circ} \mathrm{C}$. Measurement of serum $\mathrm{CgA}$ levels was performed between February and July 2005, both locally and in two reference laboratories, by a two-step IRMA (IRMA; CGA-RIA CT, CIS-bio international-Shering, Gif-sur-Yvette, France) in Venezia (ABO Association c/o Regional Center for the Study of Biological Markers of Malignancy, General Regional Hospital, Venezia, Italy) and by ELISA (DAKO Cytomation, Glostrup, Denmark) in Orbassano (Medical Oncology Unit, S. Luigi Hospital, Orbassano, Torino, Italy). Both methods were performed according to the manufacturer's instructions. All samples were assayed in duplicate by the same technician.

The IRMA assay is based on two monoclonal antibodies raised against the unprocessed central domain $\left(\mathrm{CgA}_{145-245}\right)$ of the human $\mathrm{CgA}$, allowing sensitive detection of total human $\mathrm{CgA}$. Recombinant human $\mathrm{CgA}$ was used as calibrator and the standard curve concentrations ranged from 22 to $1200 \mathrm{ng} / \mathrm{ml}$, with a minimal detectable level of $10 \mathrm{ng} / \mathrm{ml}$. Inter-assay coefficients of variation were 3.4 and $4.5 \%$ at 124.7 and $355.2 \mathrm{ng} / \mathrm{ml}$ respectively. Intra-assay coefficients of variation were 5.1, 3.0, and $7.8 \%$ for the following ranges 15-25, 90-110, and 500-700 $\mathrm{ng} / \mathrm{ml}$ respectively.

The ELISA assay is based on two polyclonal rabbit antibodies directed towards a $23 \mathrm{kDa}$ carboxylterminal fragment of human $\mathrm{CgA}$, therefore measuring more human $\mathrm{CgA}$ fragments. The calibrators were extracted from urine of patients with carcinoids and the standard curve concentrations ranged from 5 to $650 \mathrm{U} / 1$, with a minimal detectable level of $5 \mathrm{U} / 1$. Inter-assay coefficients of variation were $3.4,3.9$, and $6.8 \%$ at $11.5,52.7$, and $358 \mathrm{U} / \mathrm{l}$ respectively. Intraassay coefficients of variation were $4.5,3.8$, and $8.5 \%$ for the following ranges $5-10,15-25$, and $250-450 \mathrm{U} / \mathrm{l}$ respectively. 


\section{Statistical analysis}

$\mathrm{CgA}$ levels are reported as the mean \pm s.D., the median, and the range for both IRMA and ELISA methods. Comparisons of values from independent groups were performed using the nonparametric test of Wilcoxon. To measure the strength of association between pairs of variables without specifying dependencies, Spearman order correlations were run. A $P<0.05$ was considered significant in all tests.

In order to identify a cutoff $\mathrm{CgA}$ value for both IRMA and ELISA assays that could discriminate between controls and patients, a ROC curve was constructed using $\mathrm{CgA}$ levels from the 129 controls and those from 81 ND patients with GEP NETs, which were considered as having the disease at the moment of blood sampling. In order to identify a cutoff $\mathrm{CgA}$ value for both IRMA and ELISA assays that could discriminate between patients without and with metastases, a ROC curve was constructed using $\mathrm{CgA}$ levels from 29 patients without metastases and those from 79 metastatic patients belonging to the ND and RL groups (108 patients), all considered as having the disease at the moment of blood sampling.

ROC analysis was performed using a statistical software package (SAS, version 8.2). The area under the ROC curve (AUC) was calculated to describe the capability of the marker to discriminate between patients and controls. Sensitivity and specificity were calculated for different cutoff values. The optimal value giving the best compromise between sensitivity and specificity was chosen to analyze the performance of CgA assays in GEP NET patients. Sensitivity and specificity were calculated using the standard formulae (sensitivity $\%=$ true positive/true positive + false negative and specificity $\%=$ true negative/true negative + false positive). The correction for the disease prevalence was adopted to calculate positive predictive value (PPV) and negative predictive value (NPV): PPV (\%; probability that a positive value corresponds to a true positive result) $=$ sensitivity/sensitivity $+(1-$ specificity $) /$ prevalence of disease and NPV (\%; probability that a negative value corresponds to true negative result $)=$ specificity/specificity $+(1-$ sensitivity $) \times$ prevalence of disease.

\section{Results}

\section{CgA levels in healthy subjects and in GEP NET patients}

CgA levels, assessed by both IRMA and ELISA methods, were highly variable and not normally distributed among the 129 healthy subjects (Table 1)
Table 1 Basal chromogranin A levels in 129 healthy subjects assessed by IRMA and ELISA

\begin{tabular}{lcc}
\hline & IRMA $(\mathrm{ng} / \mathrm{ml})$ & ELISA $(\mathrm{U} / \mathrm{l})$ \\
\hline No. & 129 & 129 \\
Mean & 46.9 & 12.3 \\
S.D. & 31.2 & 10.1 \\
Median & 40.0 & 10.4 \\
Range & $17-269$ & $5-106.5$ \\
Percentile & & \\
$\quad$ 5th & 21.0 & 6.2 \\
25th & 31.0 & 8.0 \\
50th & 40.0 & 10.4 \\
75th & 54.0 & 13.7 \\
95th & 86.0 & 19.3 \\
\hline
\end{tabular}

and in the 202 GEP NET patients (Table 2). The analysis of collected data showed a good correlation between IRMA and ELISA assays in measuring $\mathrm{CgA}$ levels both in healthy subjects $(r=0.689 ; P<0.0001$; Fig. 1A) and in GEP NET patients $(r=0.848 ; P<$ 0.0001; Fig. 1B). In addition, a good correlation between local and central laboratories in measuring CgA levels both with IRMA $(r=0.846 ; P<0.0001$; Fig. 2A) and with ELISA assays $(r=0.873 ; P<$ 0.0001) was found (Fig. 2B).

\section{Diagnostic property of $\mathrm{CgA}$}

In order to identify a cutoff value that could distinguish between healthy subjects and affected patients, we performed a ROC analysis considering $\mathrm{CgA}$ levels from the 129 controls and those from 81 ND patients with GEP NETs, measured by both IRMA and ELISA assays.

As shown in Fig. 3A, the cutoff value of $53 \mathrm{ng} / \mathrm{ml}$ for the IRMA assay provided the best compromise between specificity $(71.3 \%)$ and sensitivity $(77.8 \%)$, and was chosen for further analysis. The area under the curve (AUC) was 0.834, indicating a good performance of the assay. Using this cutoff, PPV were 54 and $35 \%$ and NPV were 92 and $90 \%$ for foregut (esophagus, stomach, pancreas, and duodenum) and midgut tumors (ileum and colon) respectively. Analysis of these parameters was then performed according to the affected organ. Due to the low number of the GEP NET patients with primary site of the tumor in the duodenum, colon, and rectum, PPV and NPV were only calculated for stomach (27 and 99\%), pancreas (43 and 93\%), and ileum (29 and 92\%) respectively. $\mathrm{CgA}$ levels were below the cutoff value in 96 out of 129 normal individuals $(74.4 \%)$ and in 28 out of 45 patients $(62 \%)$ with endocrine tumors in RM. 
Table 2 Basal chromogranin A levels in 202 gastroenteropancreatic neuroendocrine tumor patients according to the recruitment group

\begin{tabular}{|c|c|c|c|c|c|c|c|c|}
\hline & \multicolumn{2}{|c|}{ New diagnosis } & \multicolumn{2}{|c|}{ Relapse } & \multicolumn{2}{|c|}{ Stable disease } & \multicolumn{2}{|c|}{ Remission } \\
\hline & $\begin{array}{c}\text { IRMA } \\
\text { (ng/ml) }\end{array}$ & ELISA (U/I) & $\begin{array}{l}\text { IRMA } \\
\text { (ng/ml) }\end{array}$ & ELISA (U/I) & $\begin{array}{l}\text { IRMA } \\
\text { (ng/ml) }\end{array}$ & ELISA (U/I) & $\begin{array}{c}\text { IRMA } \\
\text { (ng/ml) }\end{array}$ & ELISA (U/I) \\
\hline No. & 81 & 81 & 27 & 27 & 49 & 49 & 45 & 45 \\
\hline Mean & 546.1 & 349.2 & 624.6 & 364.4 & 434.6 & 160.5 & 67.3 & 19.0 \\
\hline S.D. & 1496.5 & 1020.6 & 872.8 & 639.7 & 1441.7 & 375.5 & 82.0 & 14.1 \\
\hline Median & 164 & 67 & 216 & 104 & 57 & 30 & 43 & 15 \\
\hline Range & $10-11720$ & $5-8170$ & $36-3045$ & $7-3018$ & $10-9555$ & $5-2489$ & $10-543$ & $5-66$ \\
\hline \multicolumn{9}{|c|}{ Percentile } \\
\hline 5 th & 28 & 9 & 39 & 8 & 19 & 6 & 23 & 6 \\
\hline 25th & 66 & 27 & 69 & 41 & 34 & 15 & 29 & 11 \\
\hline 50th & 164 & 67 & 216 & 104 & 57 & 30 & 43 & 15 \\
\hline 75th & 342 & 215 & 971 & 476 & 132 & 171 & 76 & 24 \\
\hline 95th & 1410 & 1423 & 3040 & 1551 & 1745 & 562 & 159 & 53 \\
\hline
\end{tabular}

As shown in Fig. 3B, the cutoff value of $16 \mathrm{U} / 1$ for the ELISA assay provided the best compromise between specificity (85.3\%) and sensitivity (84\%), and was chosen for further analysis. The AUC was 0.892, again indicating a good performance of the assay. Using this cutoff, PPV were 69 and 57\% and NPV were 93 and $96 \%$ for foregut and midgut tumors respectively. When considering the primary site of the tumor, PPV and NPV were 42 and 99\% for stomach, 60 and $94 \%$ for pancreas, and 51 and $97 \%$ for ileum respectively. $\mathrm{CgA}$ levels were below this cutoff value in 110 out of 129 normal individuals $(85.3 \%)$ and in 25 out of 45 patients $(55.6 \%)$ with endocrine tumors in RM.

Considering these cutoff levels, six healthy subjects having normal $\mathrm{CgA}$ levels by IRMA had $\mathrm{CgA}$ levels above the cutoff when assayed by ELISA. On the contrary, 20 healthy subjects having normal $\mathrm{CgA}$ by ELISA had CgA levels above the cutoff when assayed by IRMA (discordance rate $20.1 \%$ ).

\section{CgA levels in metastatic patients}

Among the 202 GEP NET patients, 123 presented with and 76 without metastases at study entry. Data concerning the presence or absence of metastases were missing in three patients. Metastatic patients had significantly $(P<0.0001)$ higher $\mathrm{CgA}$ levels than patients without metastases, both by IRMA (605.9 \pm $1537.9 \mathrm{ng} / \mathrm{ml}$, range $10-11,720 \mathrm{ng} / \mathrm{ml}$ vs $142.1 \pm$ $324.6 \mathrm{ng} / \mathrm{ml}$, range $10-2715 \mathrm{ng} / \mathrm{ml}$ ) and by ELISA assays (351.5 $\pm 899.3 \mathrm{U} / \mathrm{l}$, range $5-8170 \mathrm{U} / \mathrm{l}$ vs $47.7 \pm$ $138.9 \mathrm{U} / \mathrm{l}$, range 5-1196 U/l). However, when considering only the 108 patients with evidence of disease and without medical treatment at study entry (ND 81 and RL patients 27), the IRMA assay did not discriminate $\mathrm{CgA}$ levels of patients with metastases from those without $(676.1 \pm 1554.9 \mathrm{ng} / \mathrm{ml}$, range $10-11,270 \mathrm{ng} / \mathrm{ml}$ vs $272.9 \pm 503.6 \mathrm{ng} / \mathrm{ml}$, range 28-2715 ng/ml; $P=0.09)$. On the other hand, $\mathrm{CgA}$
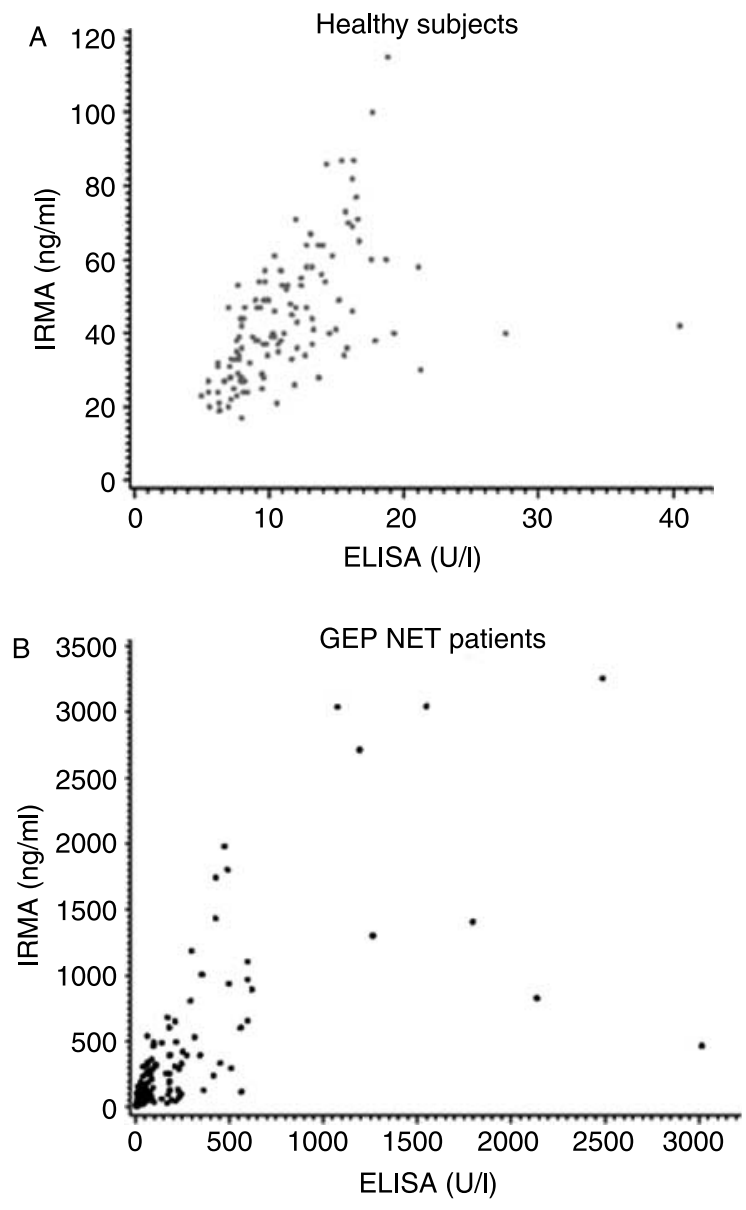

Figure 1 Comparison between ELISA and IRMA methods in 129 healthy subjects (A) and in 202 GEP NET patients (B). 

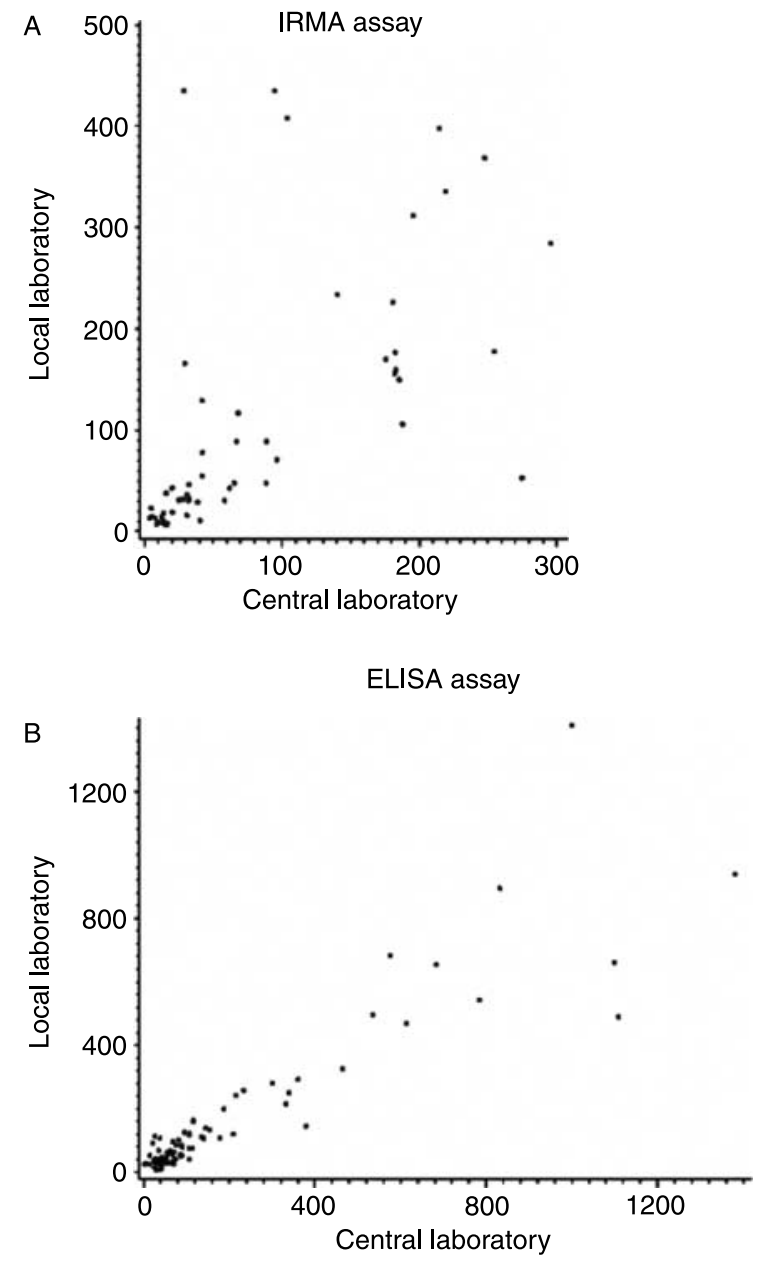

Figure 2 Comparison between local and central laboratories for IRMA (A) and ELISA (B) methods in GEP NET patients.

levels measured by the ELISA assay significantly differed in the two groups $(450.4 \pm 1073.2 \mathrm{U} / \mathrm{l}$, range $5-8170 \mathrm{U} / 1$ vs $90.2 \pm 222.4 \mathrm{U} / 1$, range $6-1196 \mathrm{U} / \mathrm{l}$; $P<0.0002$ ).

In order to identify a $\mathrm{CgA}$ cutoff value that could distinguish between patients with metastases from those without, we performed a ROC analysis evaluating $\mathrm{CgA}$ levels from affected patients $(\mathrm{ND}+\mathrm{RL}=$ 108), excluding patients in RM and those with SD. Therefore, the ROC curve was constructed by considering $\mathrm{CgA}$ levels measured by both IRMA and ELISA assays in 79 vs 29 patients with and without metastases respectively.

As shown in Fig. 4A, with the IRMA assay, the cutoff value of $146 \mathrm{ng} / \mathrm{ml}$ provided the best compromise between specificity $(55.6 \%)$ and sensitivity (57.0\%), and was chosen for further analysis. The AUC was 0.613 indicating a modest performance of

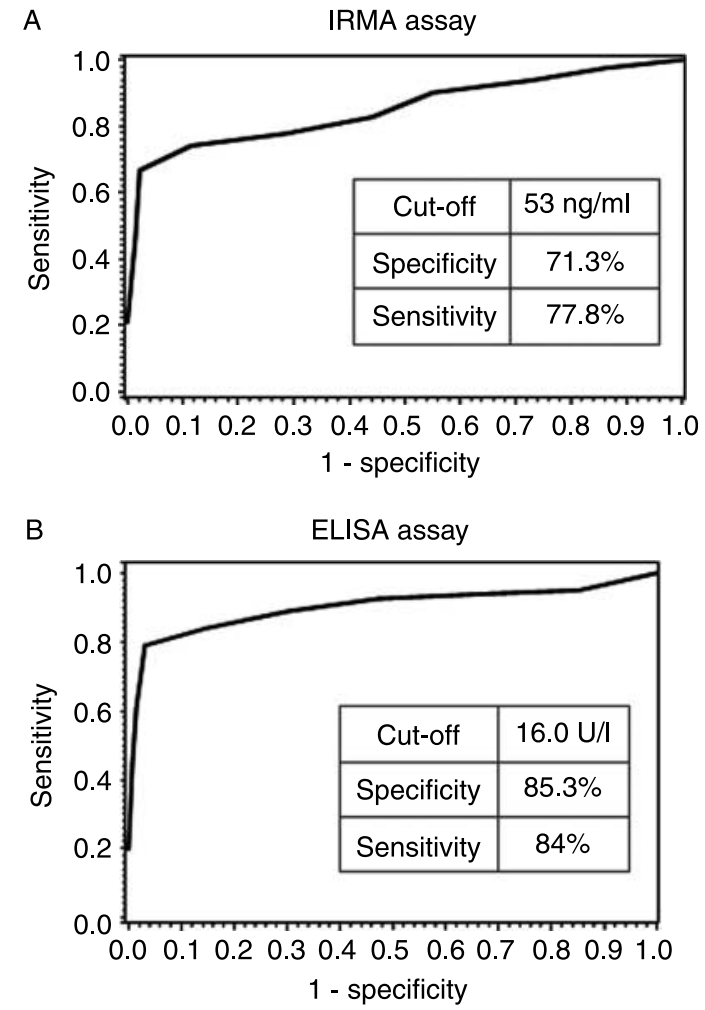

Figure 3 Receiver-operating characteristics curve obtained with 129 healthy subjects and 81 new diagnosis GEP NET patients for the IRMA (A) and for the ELISA assays (B).

the assay. Using this cutoff value, PPV were 49 and $40 \%$ and NPV were 72 and $46 \%$ for foregut and midgut tumors respectively. Analysis of these parameters was then performed according to the affected organ. Due to the low numerosity, PPV and NPV were only calculated for stomach (14 and 65\%) and pancreas (84 and $78 \%$ ) respectively.

As shown in Fig. 4B, with the ELISA assay, the cutoff value of $67.3 \mathrm{U} / 1$ provided the best compromise between specificity (71.4\%) and sensitivity (63.3\%), and was chosen for further analysis. The AUC was 0.727 , indicating again a modest performance of the assay. Using this cutoff value, PPV were 61 and 58\% and NPV were 76 and $65 \%$ for foregut and midgut tumors respectively. When considering the affected organ, it is worth to underline that for pancreatic NETs, with the chosen cutoff levels for both IRMA and ELISA assays, PPV was $84 \%$ and NPV was $78 \%$, with $90 \%$ specificity and $68 \%$ sensitivity. On the other hand, PPV and NPV for stomach were 22 and $79 \%$ with the ELISA assay.

Table 3 shows CgA levels assessed both by IRMA and ELISA assays in 79 metastatic GEP NET patients, belonging to ND and RL groups, according to the 
A

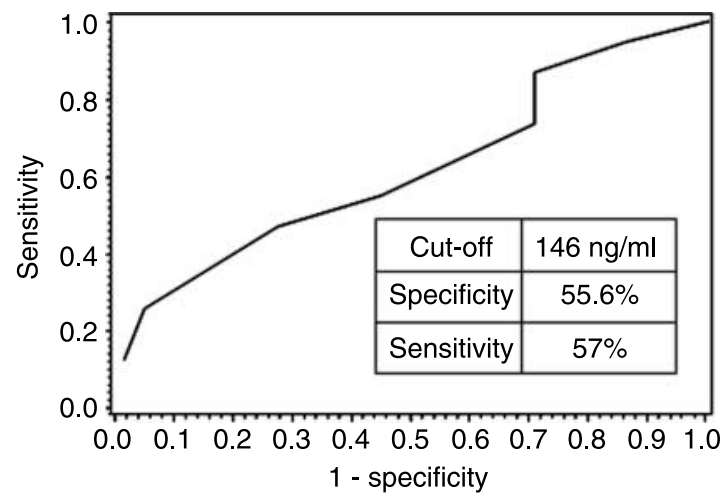

B

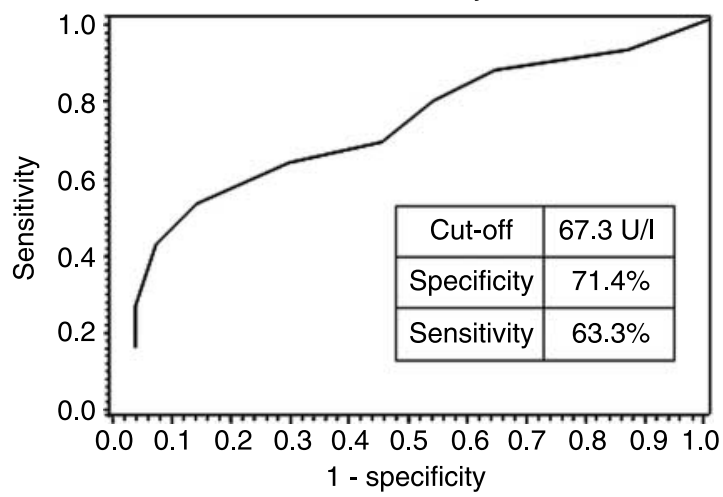

Figure 4 Receiver-operating characteristics curve obtained with 79 patients with metastases and 29 patients without metastases for the IRMA $(A)$ and for the ELISA assays (B).

spread of the disease. Data on disease extension were missing for nine patients. $\mathrm{CgA}$ levels were greater in patients with liver metastases when compared with those with locally advanced disease. In addition, $\mathrm{CgA}$ levels, evaluated by both IRMA and ELISA assays, were lower in patients with extensive metastatic spread (extra-hepatic metastases; $194.8 \pm 123.2 \mathrm{ng} / \mathrm{ml}$, range $69-423 \mathrm{ng} / \mathrm{ml} ; 81.5 \pm 70.7 \mathrm{U} / \mathrm{l}$, range $13-255 \mathrm{U} / \mathrm{l})$ than in those with liver metastases only $(800.9 \pm$ $1206.7 \mathrm{ng} / \mathrm{ml}$, range $36-4690 \mathrm{ng} / \mathrm{ml} ; \quad 515.2 \pm$ $773.2 \mathrm{U} / \mathrm{l}$, range 9-3018 U/l).

\section{Discussion}

In this multicenter observational study, we have focused on assessing the value of $\mathrm{CgA}$ as a biochemical marker for detection of GEP tumors. An important result of this study is that the employed assays for $\mathrm{CgA}$ measurement, which are the most commonly available in clinical practice, showed a very good correlation between them, in agreement with previous reports (Stridsberg et al. 2003), as well as a good correlation between the values obtained by the central laboratories in Venezia and Orbassano and those obtained by the peripheral laboratories. Therefore, we can assume that $\mathrm{CgA}$ values reported by the laboratories of the 40 Italian centers participating in the study are as reliable as those obtained in dedicated laboratories and support the reliability of $\mathrm{CgA}$ evaluation in clinical practice.

The results show that $\mathrm{CgA}$ levels are highly variable in our study population, with overlapping levels between healthy subjects, patients with active disease (ND, RL, and SD) and patients in RM, as measured by both IRMA and ELISA assays, suggesting a modest diagnostic value for $\mathrm{CgA}$ assessment in the screening procedures for GEP NETs.

The limited diagnostic power of $\mathrm{CgA}$ measurement is also underlined by the results of the ROC analysis, performed by considering healthy subjects and ND patients. The analysis indeed identified cutoff values for IRMA and ELISA assays located between the 75th and the 95th percentile of the CgA values distribution in healthy controls, with modest sensitivity (77.8 and $84 \%$ ) and specificity (71.3 and $85.3 \%$ ) for both IRMA and ELISA assays respectively. This evidence is in line with previous reports showing a relatively low diagnostic value of circulating $\mathrm{CgA}$ in NETs (Nobels et al. 1998, Tomassetti et al. 2001). This may depend on type, secretory activity, degree of neuroendocrine differentiation, and total burden of the tumors (Seregni et al. 2001), as well as on the highly variable CgA levels of the control group. Indeed, 26 and 16\% healthy subjects had high baseline CgA levels by IRMA and ELISA assays respectively, probably because of the many potential tissue sources of the peptide (Lamberts et al. 2001). Furthermore, chronic atrophic gastritis, which causes high-circulating CgA levels (Syversen et al. 2004), was not completely ruled out in our control group, even if all healthy subjects were asymptomatic.

The CgA cutoff levels identified by the ROC analysis in the present study are lower than those described in previous studies. Stridsberg et al. (2003) adopted the kit cutoff levels without validating them and considered a patients group including subjects who lacked signs of NET. Other authors calculated the cutoff levels on the basis of control groups lacking strict exclusion criteria (Ferrari et al. 2004, Nehar et al. 2004) or previously diagnosed with non-GEP NETs (Ferrari et al. 2004).

The PPV and NPV of CgA measurement for both IRMA and ELISA were calculated on the basis of the disease prevalence in our study group. Reliable epidemiological data concerning GEP NET, essential 
Table 3 Basal chromogranin A levels in $79^{a}$ gastroenteropancreatic neuroendocrine tumor patients according to metastatic spread

\begin{tabular}{|c|c|c|c|c|c|c|}
\hline & \multicolumn{2}{|c|}{ Locally advanced disease } & \multicolumn{2}{|c|}{ Liver metastases } & \multicolumn{2}{|c|}{$\begin{array}{l}\text { Liver and extra-hepatic } \\
\text { metastases }\end{array}$} \\
\hline & IRMA (ng/ml) & ELISA (U/I) & IRMA (ng/ml) & ELISA (U/I) & IRMA (ng/ml) & ELISA (U/I) \\
\hline No. & 22 & 22 & 39 & 39 & 9 & 9 \\
\hline Mean & 359.7 & 256.8 & 800.9 & 515.2 & 194.8 & 81.5 \\
\hline S.D. & 412.8 & 449.4 & 1206.7 & 773.2 & 123.2 & 70.7 \\
\hline Median & 199 & 65 & 327 & 219 & 146 & 60 \\
\hline Range & $22-1410$ & $5-1798$ & $10-4990$ & $8-3300$ & $69-423$ & $13-255$ \\
\hline \multicolumn{7}{|c|}{ Percentile } \\
\hline 5 th & 27 & 5 & 36 & 9 & 69 & 13 \\
\hline 25th & 44 & 28 & 103 & 95 & 107 & 42 \\
\hline 50th & 199 & 65 & 327 & 219 & 146 & 60 \\
\hline 75th & 491 & 234 & 971 & 562 & 291 & 89 \\
\hline 95th & 1305 & 1266 & 4690 & 3018 & 423 & 255 \\
\hline
\end{tabular}

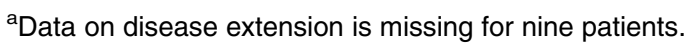

to accurately identify PPV and NPV for CgA levels prevalence in Italy, are currently lacking. Therefore, correction for the disease prevalence in the general population was not performed. As a consequence, data analysis overestimates PPVs and underestimates NPVs, probably impairing the diagnostic value of these parameters. Our analysis shows quite low PPVs, therefore suggesting even lower PPVs when considering disease prevalence in the general population. Thus, the identified PPVs for CgA assessment cannot be considered reliable discriminators for disease presence. On the other hand, our analysis shows very high NPVs, therefore suggesting even higher NPVs when considering disease prevalence in the general population. Thus, identified NPVs for CgA assessment could be considered reliable discriminators for disease absence.

Twenty-eight out of 202 patients (13.9\%) were classified differently by the two assays, suggesting that $\mathrm{CgA}$ assessment with only one out of the two assays is not sufficient to exclude the presence of increased $\mathrm{CgA}$ levels in these patients. However, the discordance rate observed in our database is much lower than that reported by Ferrari et al. (2004). We previously demonstrated that the discordance between the results of the two assays is not due to the use of different blood derivatives (Leon et al. 2005), but might be due to the different ability of the antibodies to detect $\mathrm{CgA}$ derived peptides. Moreover, these findings support the hypothesis that the two $\mathrm{CgA}$ kits used may provide different information, since a $20.1 \%$ discordance rate was also found among healthy subjects.

In keeping with previous studies (Nehar et al. 2004), we found higher CgA levels in metastatic patients when compared with those without metastases.
However, our study does not demonstrate a statistically significant difference among patient groups with increasing metastatic spread as indicated by previous studies (Nobels et al. 1998, Peracchi et al. 2003). On the contrary, we found that $\mathrm{CgA}$ levels assayed with both methods were lower in patients with very extensive metastatic spread when compared with those having metastases limited to the liver. The influence of concomitant therapies can be excluded, since the analysis was performed on newly diagnosed patients, neither previously treated by surgery nor by medical therapy. Therefore, the lower CgA levels in patients with very extensive metastatic spread might be attributed to a possible loss of neuroendocrine differentiation, probably indicating a more aggressive behavior. It has been previously demonstrated that $\mathrm{CgA}$ is normally absent or only focally expressed in poorly differentiated endocrine carcinomas (Rindi \& Klöppel 2004). However, the lack of complete information concerning proliferative index and histology in these tumors does not allow us to draw any definitive conclusion. Follow-up data are needed to better clarify this issue, also in the light of previous studies showing that elevated $\mathrm{CgA}$ levels are strongly correlated with tumor volume (Nobels et al. 1997) and disease extent (Seregni et al. 2001).

The ROC analysis identified a cutoff level of $146 \mathrm{ng} / \mathrm{ml}$ for the IRMA and of $67.3 \mathrm{U} / \mathrm{l}$ for the ELISA assays as discriminating between patients with metastases and those without, but sensitivity (57 and $63.3 \%$ respectively) and specificity (55.6 and $71.4 \%$ respectively) were quite low. On the other hand, the calculated NPVs of CgA measurement for both IRMA and ELISA assays are very high, suggesting that $\mathrm{CgA}$ 
values below the identified threshold levels are highly indicative of the absence of metastases in foregut and midgut GEP NET patients. Correction for the prevalence of the disease in the general population would again result in higher NPVs, suggesting that the CgA cutoff levels of $146 \mathrm{ng} / \mathrm{ml}$ for IRMA and $67.3 \mathrm{U} / 1$ for ELISA could discriminate patients without metastases from metastatic patients. On the other hand, our analysis showed rather low PPVs for discriminating metastatic patients, indicating that values above the chosen cutoff levels are not predictive of the presence of metastases in the majority of cases, also because they are very likely overestimates. However, our data also point out that in patients with pancreatic tumors CgA levels, evaluated with both methods, have a high predictive value for the presence of metastases, since they correctly identify $84 \%$ of these patients as having metastatic disease, with good specificity (90\%), but modest sensitivity $(68 \%)$. These data suggest that in patients diagnosed with pancreatic NETs CgA evaluation might be useful to identify patients in which local and distant metastases should be looked for by an accurate clinical evaluation. This issue is important, since it has been previously demonstrated that in pancreatic NETs, the presence of metastases profoundly influences survival rate, which is significantly better in patients without metastases (Madeira et al. 1998, Chu et al. 2002, Gullo et al. 2003, Panzuto et al. 2005, Tomassetti et al. 2005). Moreover, the 5-year survival rate was reported to be $60-100 \%$ for localized disease, $40 \%$ for regional disease, $29 \%$ for distant metastases, and $80 \%$ for all stages (Eriksson et al. 1990, Modlin et al. 2003). Therefore, $\mathrm{CgA}$ evaluation could have a clinical value also for prognosis.

The study presented here also found for ELISA a higher sensitivity and specificity (84 and 85\% respectively) when compared with IRMA assay (71.3 and $77.8 \%$ respectively) in identifying patients affected by NETs. The greater ELISA sensitivity might be due to a more extensive CgA cleavage by GEP NETs. Indeed, the IRMA assay mainly evaluates intact molecules and major $\mathrm{CgA}$ fragments, since it employs two antibodies recognizing the central part of human $\mathrm{CgA}$, which is unexposed to proteolysis (Degorce et al. 1999, Bernini et al. 2001). In pathological conditions, such as NETs, different proteolytic processes may take place, generating a variable number of fragments (Taupenot et al. 2003), which are better assessed by the ELISA assay. However, further studies are needed to address the specific $\mathrm{CgA}$ cleavage in different tumors. Initial proteomic studies have identified 11 novel $\mathrm{CgA}$-derived peptides in endocrine tumors, supporting the hypothesis that different tumors may process differently the entire molecule, representing a possible specific signature (Orr et al. 2002).

In conclusion, our study shows that an accurate comparison between healthy subjects and GEP NET patients does not provide cutoff levels that could discriminate between the two groups with a sensitivity and a specificity high enough to demonstrate $\mathrm{CgA}$ as an efficient biochemical marker in the diagnostic screening of GEP NET. These data indicate that CgA serum levels can be helpful for the clinical management of NETs, but with low sensitivity and specificity for diagnostic purposes. On the other hand, the main utility of $\mathrm{CgA}$ measurement may be in patient monitoring. Therefore, follow-up prospective data are necessary to examine the performance of $\mathrm{CgA}$ assessment in evaluating follow-up and treatment efficacy in GEP NET patients. Further studies are ongoing to clarify this issue.

\section{Acknowledgements}

The authors would like to thank the Business Unit Oncology of Novartis Farma S.p.A. (Italy), in particular Dr Francesco Bartucci, for the study support, and Dr Paola Vaghi from OPIS (Italy) for her statistical assistance in the data management. The following participating members of the 'Italian CROMaNET Working Group' with their institution listed in parenthesis, which have enrolled patients in the study are: Oscar Alabiso (Ospedale S Maria Maggiore della Carità, Novara), Fabrizio Artioli (Ospedale B Ramazzini/Az.USL, Carpi), Oscar Bertetto (Ospedale Le Molinette, Torino), Franco Grimaldi (Azienda Ospedaliera S Maria della Misericordia, Udine), Laura Tomasello (Istituto Nazionale Per la Ricerca sul Cancro IST, Genova), Cristian Massacesi (Ospedali Riuniti Umberto I-Salesi-Lancisi, Ancona), Giorgio Arnaldi (Azienda Ospedaliera Umberto I, Ancona), Mario Botta (ASL 21 Ospedale S Spirito, Casale Monferrato), Vincenzo Iaffaioli (Fondazione G Pascale, Napoli), Renato Cannizzaro, (Centro di Riferimento Oncologico-CRO, Aviano), Antonio Calabrò (Ospedale Careggi, Firenze), Donatella Zamagni (Ospedale C Poma, Mantova), Vincenzo Caragliano (Ospedale Vittorio Emanuele II, Catania), Giacomo Cartenì (Ospedale Cardarelli, Napoli), Antonio Contu (Ospedale Civile, Sassari), Modesto D’Aprile (Ospedale S Maria Goretti, Latina), Claudio De Angelis Ospedale Le Molinette, Torino), Nicola Fazio (Istituto Oncologico Europeo, Milano), Ettore degli Uberti (Università degli Studi di Ferrara, 
Ferrara), Gianfranco Delle Fave (Ospedale S Andrea, Roma), Maria Pia Brizzi (Azienda Ospedaliera S Luigi, Orbassano), Alfredo Falcone (Ospedale Civile, Livorno), Guido Francini (Policlinico Le Scotte, Siena), Ciro Guerriero (Azienda Ospedaliera Moscati, Monteforte Irpino), Silvio Monfardini (Ospedale Busonera, Padova), Stefano Iacobelli (Policlinico SS Annunziata, Chieti), Gabriele Luppi (Centro Oncologico Modenese, Modena), Bruno Massidda (Policlinico Universitario, Monserrato, Cagliari), Francesco Minuto (Università di Genova, DISEM, Genova), Graziella Pinotti (Ospedale di Circolo e Fondaz, Macchiù, Varese), Paolo Pederzoli (Policlinico G B Rossi, Verona), Sergio Ricci (Ospedale S Chiara, Pisa), Salvatore Siena (Ospedale Niguarda Cà Granda, Milano), Nicola Sicolo (Azienda Ospedaliera Di Padova, Padova), Davide Campana (Policlinico S Orsola Malpighi, Bologna), Roberto Valcavi (Arcispedale S Maria Nuova, Reggio Emilia), Lucia Tozzi (Ospedale Casa Sollievo della Sofferenza, S Giovanni Rotondo), Valter Vincenti (Ospedale Civile, Belluno), Francesco Di Vito (Ospedale Aosta, Aosta), and Salvatore Tumolo (Ospedale di Pordenone, Pordenone). The authors declare that there is no conflict of interest that would prejudice the impartiality of this scientific work.

\section{References}

Baudin E, Bidart JM, Bachelot A, Ducreux M, Elias D, Ruffié P \& Schlumberger M 2001 Impact of chromogranin A measurement in the work-up of neuroendocrine tumors. Annals of Oncology 12 S79-S82.

Bernini GP, Moretti A, Ferdeghini M, Ricci S, Letizia C, D’Erasmo E, Argenio GF, Argenio GF \& Salvetti A 2001 A new human chromogranin 'A' immunoradiometric assay for the diagnosis of neuroendocrine tumors. British Journal of Cancer 84 636-642.

Canale MP \& Bravo EL 1994 Diagnosis specificity of serum chromogranin-A for pheochromocytoma in patients with renal dysfunction. Journal of Clinical Endocrinology and Metabolism 78 1139-1144.

Chu QD, Hill HC, Douglass HO Jr, Driscoll D, Smith JL, Nava HR \& Gibbs JF 2002 Predictive factors associated with long-term survival in patients with neuroendocrine tumors of the pancreas. Annals of Surgical Oncology 9 855-862.

Degorce F, Goumon Y, Jacquemart L, Vidaud C, Bellanger L, Pons-Anicet D, Seguin P, Metz-Boutigue MH \& Aunis D 1999 A new human chromogranin A (CGA) immunoradiometric assay involving monoclonal antibodies raised against the unprocessed central domain. British Journal of Cancer 79 65-71.
Eriksson B, Arnberg H, Lindgren PG, Lorelius LE, Magnusson A, Lundqvist G, Skogseid B, Wide L, Wilander E \& Oberg K 1990 Neuroendocrine pancreatic tumours: clinical presentations, biochemical and histopathological findings in 84 patients. Journal of Internal Medicine 228 103-113.

Ferrari L, Seregni E, Lucignani G, Bajetta E, Martinetti A, Aliberti G, Pallotti F, Procopio G, Della Torre S, Luksch R et al. 2004 Accuracy and clinical correlates of two different methods for chromogranin A assay in neuroendocrine tumors. International Journal of Biological Markers 19 295-304.

Guignat L, Bidart JM, Nocera M, Comoy E, Schlumberger M $\&$ Baudin E 2001 Chromogranin A and the $\alpha$-subunit of glycoprotein hormones in medullary thyroid carcinoma and phaeochromocytoma. British Journal of Cancer $\mathbf{8 4}$ 808-812.

Gullo L, Migliori M, Falconi M, Pederzoli P, Bettini R, Casadei R, Delle Fave G, Corleto VD, Ceccarelli C, Santini D et al. 2003 Nonfunctioning pancreatic endocrine tumors: a multicenter clinical study. American Journal of Gastroenterology 98 2435-2439.

Lamberts SW, Hofland LJ \& Nobels FR 2001 Neuroendocrine tumor markers. Frontiers in Neuroendocrinology 22 309-339.

Leon A, Torta M, Dittadi R, degli Uberti E, Ambrosio MR, Delle Fave G, De Braud F, Tomassetti P, Gion M \& Dogliotti L 2005 Comparison between two methods in the determination of circulating chromogranin A in neuroendocrine tumors (NETs): results of a prospective multicenter observational study. International Journal of Biological Markers 20 156-168.

Madeira I, Terris B, Voss M, Denys A, Sauvanet A, Flejou JF, Vilgrain V, Belghiti J, Bernades P \& Ruszniewski P 1998 Prognostic factors in patients with endocrine tumours of the duodenopancreatic area. Gut 43 422-427.

Modlin IM, Lye KD \& Kidd M 2003 A 5-decade analysis of 13715 carcinoid tumors. Cancer 97 934-939.

Nehar D, Lombard-Bohas C, Olivieri S, Claustrat B, Chayvialle JA, Penes MC, Sassolas G \& Borson-Chazot F 2004 Interest of chromogranin A for diagnosis and follow-up of endocrine tumours. Clinical Endocrinology 60 644-652.

Nobels FRE, Kwekkeboom DJ, Coopmans W, Schoenmakers CHH, Lindemans J, De Herder WW, Krenning EP, Bouillon R \& Lamberts SWJ 1997 Chromogranin A as serum marker for neuroendocrine neoplasia: comparison with neuron-specific enolase and the $\alpha$-subunit secreting of glycoprotein hormones. Journal of Clinical Endocrinology and Metabolism 82 2622-2628.

Nobels FRE, Kwekkeboom DJ, Boiullon R \& Lamberts SW 1998 Chromogranin A: its clinical value as markers of neuroendocrine tumours. European Journal of Clinical Investigation 28 431-440. 
Orr DF, Chen T, Johnsen AH, Chalk R, Buchanan KD, Sloan JM, Rao P \& Shaw C 2002 The spectrum of endogenous human chromogranin A-derived peptides identified using a modified proteomic strategy. Proteomics 2 1586-1600.

Panzuto F, Nasoni S, Falconi M, Corleto VD, Capurso G, Cassetta S, Di Fonzo M, Tornatore V, Milione M, Angeletti S et al. 2005 Prognostic factors and survival in endocrine tumor patients: comparison between gastrointestinal and pancreatic localization. Endocrine Related Cancer 12 1083-1092.

Peracchi M, Conte D, Gebbia C, Penati C, Pizzinelli S, Arosio M, Corbetta S \& Spada A 2003 Plasma chromogranin A in patients with sporadic gastro-entero-pancreatic neuroendocrine tumors or multiple endocrine neoplasia type 1 . European Journal of Endocrinology 148 39-43.

Rindi G \& Klöppel G 2004 Endocrine tumors of the gut and pancreas tumor biology and classification. Neuroendocrinology 80 12-15.

Schürmann G, Raeth U, Wiedenmann B, Buhr H \& Herfarth C 1992 Serum chromogranin A in the diagnosis and the follow-up of neuroendocrine tumors of the gastroenteropancreatic tract. World Journal of Surgery 16 697-702.

Seregni E, Ferrari L, Bajetta E, Martinetti A \& Bombardieri E 2001 Clinical significance of blood chromogranin A measurement in neuroendocrine tumours. Annals of Oncology 12 S69-S72.

Stivanello M, Berruti A, Torta M, Termine A, Tampellini M, Gorzegno G, Angeli A \& Dogliotti L 2001 Circulating chromogranin A in the assessment of patients with neuroendocrine tumours. A single institution experience. Annals of Oncology 12 S73-S77.

Stridsberg M, Öberg K, Li Q, Engström U \& Lundqvist G 1995 Measurements of chromogranin A, chromogranin B (secretogranin I), chromogranin C, (secretogranin II) and pancreastatin in plasma and urine from patients with carcinoid tumours and endocrine pancreatic tumours. Journal of Endocrinology 144 49-59.

Stridsberg M, Eriksson B, Öberg K \& Janson ET 2003 A comparison between three commercial kits for chromogranin A measurements. Journal of Endocrinology 177 337-341.

Syversen U, Ramsrad H, Gamme K, Qvigstad G, Falkmer S \& Waldum HL 2004 Clinical significance of elevated serum chromogranin A levels. Scandinavian Journal of Gastroenterology 39 969-973.

Taupenot L, Harper KL \& O'Connor DT 2003 The chromogranin-secretogranin family. New England Journal of Medicine 348 1134-1149.

Tomassetti P, Migliori M, Simoni P, Casadei R, De Iasio R, Corinaldesi R \& Gullo L 2001 Diagnostic value of plasma chromogranin A in neuroendocrine tumours. European Journal of Gastroenterology and Hepatology 13 55-58.

Tomassetti P, Campana D, Piscitelli L, Casadei R, Santini D, Nori F, Morselli-Labate AM, Pezzulli R \& Corinaldesi R 2005 Endocrine pancreatic tumors: factors correlated with survival. Annals of Oncology 16 1806-1810. 